\title{
Nucleon $\Sigma$ Term in the Chiral Mixing Approach
}

\author{
Veljko Dmitrašinović* \\ Institute of Physics, Belgrade University, \\ Pregrevica 118, Zemun, P.O.Box 57, 11080 Beograd, Serbia \\ E-mail: dmitrasindipb.ac.rs
}

\section{Hua-Xing Chen}

School of Physics and Nuclear Energy Engineering and International Research Center for

Nuclei and Particles in the Cosmos, Beihang University, Beijing 100191, China

E-mail: Lxchendouaa.edu.cn

\section{Atsushi Hosaka}

Research Center for Nuclear Physics, Osaka University, Ibaraki 567-0047, Japan

E-mail: hosakadrcnp.osaka-u.ac.jp

We calculate the $\Sigma_{\pi N}$ term in the chiral mixing approach to baryons, i.e., with $S U_{L}(3) \times S U_{R}(3)$ chiral multiplets $[(\mathbf{6}, \mathbf{3}) \oplus(\mathbf{3}, \mathbf{6})],[\mathbf{3}, \overline{\mathbf{3}}) \oplus(\overline{\mathbf{3}}, \mathbf{3})]$ and $[(\overline{\mathbf{3}}, \mathbf{3}) \oplus(\mathbf{3}, \overline{\mathbf{3}})]$, admixed in the baryons, using known constraints on the current quark masses $m_{u}^{0}, m_{d}^{0}$. We show that the $[(\mathbf{6}, \mathbf{3}) \oplus(\mathbf{3}, \mathbf{6})]$ multiplet makes a contribution enhanced by a factor of $\frac{57}{9} \simeq 6.33$, (purely due to $S U_{L}(2) \times S U_{R}(2)$ algebra) that leads to $\Sigma_{\pi N} \geq\left(1+\frac{48}{9} \sin ^{2} \theta\right) \frac{3}{2}\left(m_{u}^{0}+m_{d}^{0}\right)=60 \mathrm{MeV}$, in general accord with "experimental" values of $\Sigma_{\pi N}$. The chiral mixing angle $\theta$ is given by $\sin ^{2} \theta=\frac{3}{8}\left(g_{A}^{(0)}+g_{A}^{(3)}\right)$, where $g_{A}^{(0)}=0.33 \pm$ 0.08 , or $0.28 \pm 0.16$, and $g_{A}^{(3)}=1.267$, are the flavor singlet and third component of the octet axial couplings. These results show that there is no need for $q^{4} \bar{q}$ components, and in particular, no need for an $s \bar{s}$ component in the nucleon.

XV International Conference on Hadron Spectroscopy-Hadron 2013

4-8 November 2013

Nara, Japan

\footnotetext{
* Speaker.
} 


\section{Introduction}

For more than a quarter-century any deviation of the nucleon $\Sigma_{\pi N}$ term extracted from the measured $\pi N$ scattering partial wave analyses (in the following to be called "measured value", for brevity) from $25 \mathrm{MeV}$ was interpreted as an increase of Zweig-rule-breaking in the nucleon, or equivalently to an increased $s \bar{s}$ content $y=\frac{2\langle N|\bar{s}| N\rangle}{\langle N|\bar{u} u+\bar{u} u| N\rangle}$ of the nucleon, Refs. [四, ■, []]. As all "measurements" of $\Sigma_{\pi N}$ have yielded values ranging from $55 \mathrm{MeV}$ to $75 \mathrm{MeV}$ [四], that are substantially larger than the expected $25 \mathrm{MeV}$, it has consequently appeared that the $s \bar{s}$ content of the nucleon must be (very) large.

A number of experiments have measured the $s \bar{s}$ contributions to nucleon observables other than the $\Sigma_{\pi N}$ term [ [1] . Not one of them has found a result larger than a few $\%$ of the $u$ (and/or $\bar{u}$ ) and $d$ (and/or $\bar{d}$ ) contributions, ${ }^{1}$ thus making the $s \bar{s}$ content of the nucleon effectively negligible $y \simeq 0$. Thus, the enigma has deepened: how is it possible to have such a large $\Sigma_{\pi N}$ term without any $s \bar{s}$ content in other observables? In the meantime the nucleon $\Sigma_{\pi N}$ term has been shown as an important ingredient in searches for (supersymmetric) cold dark matter, Ref. [ [ $]$ ] and in the QCD phase diagram, thereby only increasing the stakes.

In this report we show explicitly an alternative mechanism of hadronic $\Sigma_{\pi N}$ term enhancement with strangeness content $y=0$ and pin-point the source of the enhancement to the $(6,3)=[(\mathbf{6}, \mathbf{3}) \oplus$ $(\mathbf{3}, \mathbf{6})] \rightarrow\left(1, \frac{1}{2}\right)=\left[\left(\mathbf{1}, \frac{\mathbf{1}}{\mathbf{2}}\right) \oplus\left(\frac{\mathbf{1}}{\mathbf{2}}, \mathbf{1}\right)\right]$ chiral component (in the $S U_{L}(3) \times S U_{R}(3)$ and $S U_{L}(2) \times S U_{R}(2)$ notations, respectively) of the nucleon. This component contributes about three quarters of the enhanced value of $\Sigma_{\pi N} \geq 55 \mathrm{MeV}$, which would otherwise be $\geq 14 \mathrm{MeV}$, while keeping a vanishing $s \bar{s}$ component in the nucleon. The same $\left(1, \frac{1}{2}\right)$ chiral component is crucial for the proper description of the nucleon's isovector axial coupling $g_{A}^{(3)}=1.267$.

We show in some detail how the $\Sigma_{\pi N}$ term enhancement emerges from the $S U_{L}(2) \times S U_{R}(2)$ chiral algebra. To that end we use a hadronic two-flavor $S U_{L}(2) \times S U_{R}(2)$ chiral mixing model, in which the $s \bar{s}$ content of the nucleon vanishes, $y=0$, per definitionem. Baryons in the spontaneously broken symmetry phase may be effectively described by a few chiral components: it was shown in

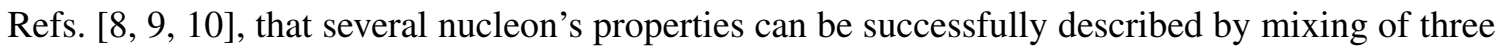
chiral multiplet components. Of the two viable chiral mixing scenarios, only the Harari one [Q, [0], described by

$$
|N\rangle=\sin \theta|(6,3)\rangle+\cos \theta(\cos \varphi|(3, \overline{3})\rangle+\sin \varphi|(\overline{3}, 3)\rangle),
$$

has survived the inclusion of the baryons' anomalous magnetic moments in the three-flavor case [ए]]. Here we use the original $S U_{L}(3) \times S U_{R}(3)$ notation, so as to distinguish between the two kinds of $\left(\frac{1}{2}, 0\right)$ multiplets in $S U_{L}(2) \times S U_{R}(2)$, though we shall need only the two-flavor version.

\section{Calculation}

To calculate the nucleon $\Sigma_{\pi N}$ term, we use the $\Sigma$ operator defined as the double commutator

$$
\Sigma=\frac{1}{3} \delta^{a b}\left[Q_{5}^{a},\left[Q_{5}^{b}, H_{\chi \mathrm{SB}}\right]\right],
$$

\footnotetext{
${ }^{1}$ This makes these effects compatible with the (much more) mundane isospin-violating corrections, from which they are indistinguishable [目].
} 
of the axial charges $Q_{5}^{a}$ and the chiral symmetry breaking Hamiltonian $H_{\chi S B}{ }^{2}$. It was introduced by Dashen [[13] as a way of separating out the explicit chiral $S U_{L}(2) \times S U_{R}(2)$ symmetry breaking part $H_{\chi S B}$ from the total Hamiltonian. Its (diagonal) nucleon matrix element $\Sigma_{\pi N}=\langle N|\Sigma| N\rangle$ determines the shift of the nucleon mass due to the chiral symmetry breaking current quark masses [[13], [4]]. Ensuring that the (spontaneously broken) chiral symmetry is properly implemented is particularly

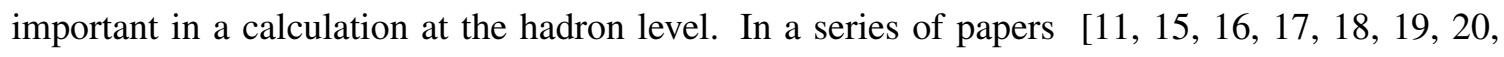
[2]] we have developed a (linear realization) chiral Lagrangian that reproduces the results of the phenomenological chiral mixing method.

We follow Ref. [[2], and use an explicit $\chi$ SB "bare" nucleon mass and the corresponding $\chi \mathrm{SB}$ Hamiltonian density

$$
\mathscr{H}_{\chi S B}^{\mathrm{N}}=\sum_{i=1}^{3} \bar{N}_{i} M_{N_{i}}^{0} N_{i}+\bar{\Delta}_{\left(1, \frac{1}{2}\right)} M_{\Delta\left(1, \frac{1}{2}\right)}^{0} \Delta_{\left(1, \frac{1}{2}\right)}
$$

where $i$ stands for the three chiral multiplets $\left(1, \frac{1}{2}\right),\left(\frac{1}{2}, 0\right)$ and $\left(0, \frac{1}{2}\right)$. A priori, we do not know the values of the "current" nucleon masses, except for a lower limit: they cannot be smaller than three isospin-averaged current quark masses: $M_{N_{i}}^{0} \geq 3 \bar{m}_{q}^{0}=\frac{3}{2}\left(m_{u}^{0}+m_{d}^{0}\right)$. For simplicity's sake, we shall assume, as a first approximation, that all three chiral components have the same "current" nucleon $\operatorname{mass} M_{N}^{0}=M_{N(6,3)}^{0}=M_{N\left(1, \frac{1}{2}\right)}^{0}=M_{\Delta\left(1, \frac{1}{2}\right)}^{0}=M_{(3, \overline{3})}^{0}=M_{\left(\frac{1}{2}, 0\right)}^{0}=M_{(\overline{3}, 3)}^{0}=M_{\left(0, \frac{1}{2}\right)}^{0}=\frac{3}{2}\left(m_{u}^{0}+m_{d}^{0}\right)$.

The chiral $S U_{L}(2) \times S U_{R}(2)$ generators $Q_{5}^{a}$ and their commutators with fields were worked out in Refs. [16, 미, ㅁ8, ए9]:

$$
\begin{aligned}
& {\left[Q_{5}^{a}, N_{\left(1, \frac{1}{2}\right)}\right]=\gamma_{5}\left(\frac{5}{3} \frac{\tau^{a}}{2} N_{\left(1, \frac{1}{2}\right)}+\frac{2}{\sqrt{3}} T^{a} \Delta_{\left(1, \frac{1}{2}\right)}\right),} \\
& {\left[Q_{5}^{a}, \Delta_{\left(1, \frac{1}{2}\right)}\right]=\gamma_{5}\left(\frac{2}{\sqrt{3}} T^{\dagger a} N_{\left(1, \frac{1}{2}\right)}+\frac{1}{3} t_{(3 / 2)}^{a} \Delta_{\left(1, \frac{1}{2}\right)}\right),} \\
& {\left[Q_{5}^{a}, N_{\left(\frac{1}{2}, 0\right)}\right]=\gamma_{5} \frac{\tau^{a}}{2} N_{\left(\frac{1}{2}, 0\right)},} \\
& {\left[Q_{5}^{a}, N_{\left(0, \frac{1}{2}\right)}\right]=-\gamma_{5} \frac{\tau^{a}}{2} N_{\left(0, \frac{1}{2}\right)},}
\end{aligned}
$$

where $a=1,2,3, t_{\left(\frac{3}{2}\right)}^{i}$ are the isospin- $\frac{3}{2}$ generators of the $S U(2)$ group and $T^{i}$ are the so-called iso-spurion $(2 \times 4)$ matrices, with the following properties (see Appendix B of Ref. [एष]])

$$
\begin{aligned}
T^{i \dagger} T^{k} & =\frac{3}{4} \delta^{i k}-\frac{1}{6}\left\{t_{\left(\frac{3}{2}\right)}^{i}, t_{\left(\frac{3}{2}\right)}^{j}\right\}+\frac{i}{3} \varepsilon^{i j k} t_{\left(\frac{3}{2}\right)}^{k}, \\
T^{i} T^{k \dagger} & =P_{\frac{3}{2}}^{i k} .
\end{aligned}
$$

The chiral $S U_{L}(2) \times S U_{R}(2)$ double commutators for the $\left(1, \frac{1}{2}\right)$ and $\left(\frac{1}{2}, 1\right)$ chiral multiplet are

$$
\left[Q_{5}^{b},\left[Q_{5}^{a}, \bar{N}_{\left(1, \frac{1}{2}\right)} N_{\left(1, \frac{1}{2}\right)}\right]\right]=\frac{41}{9} \delta^{a b} \bar{N}_{\left(1, \frac{1}{2}\right)} N_{\left(1, \frac{1}{2}\right)}+\bar{\Delta}_{\left(1, \frac{1}{2}\right)}\left(2 \delta^{a b}-\frac{4}{9}\left\{t_{\left(\frac{3}{2}\right)}^{a}, t_{\left(\frac{3}{2}\right)}^{b}\right\}\right) \Delta_{\left(1, \frac{1}{2}\right)}+\ldots
$$

where $\ldots$ stand for the off-diagonal terms, such as $\bar{N}_{\left(1, \frac{1}{2}\right)}(\ldots) \Delta_{\left(1, \frac{1}{2}\right)}$, and their Hermitian conjugates.

\footnotetext{
${ }^{2}$ For normalization and notational conventions see Ref. [미].
} 
We contract Eq. (R.4) with $\frac{1}{3} \delta^{a b}$ (where summation over repeated indices is understood) to find:

$$
\frac{1}{3} \delta^{a b}\left[Q_{5}^{b},\left[Q_{5}^{a}, \bar{N}_{\left(1, \frac{1}{2}\right)} N_{\left(1, \frac{1}{2}\right)}\right]\right]=\frac{41}{9} \bar{N}_{\left(1, \frac{1}{2}\right)} N_{\left(1, \frac{1}{2}\right)}+\frac{8}{9} \bar{\Delta}_{\left(1, \frac{1}{2}\right)} \Delta_{\left(1, \frac{1}{2}\right)}+\ldots
$$

where we have used the identity $t_{\left(\frac{3}{2}\right)}^{a} t_{\left(\frac{3}{2}\right)}^{a}=\frac{15}{4} \mathbf{1}_{4 \times 4}$, and similarly for the $\Delta$-field contribution

$$
\frac{1}{3} \delta^{a b}\left[Q_{5}^{b},\left[Q_{5}^{a}, \bar{\Delta}_{\left(1, \frac{1}{2}\right)} \Delta_{\left(1, \frac{1}{2}\right)}\right]\right]=\frac{16}{9} \bar{N}_{\left(1, \frac{1}{2}\right)} N_{\left(1, \frac{1}{2}\right)}+\frac{13}{9} \bar{\Delta}_{\left(1, \frac{1}{2}\right)} \Delta_{\left(1, \frac{1}{2}\right)}+\ldots
$$

This finally leads to

$$
\Sigma_{\pi N}=\sin ^{2} \theta\left(\frac{41}{9} M_{N\left(1, \frac{1}{2}\right)}^{0}+\frac{16}{9} M_{\Delta\left(1, \frac{1}{2}\right)}^{0}\right)+\cos ^{2} \theta\left(\cos ^{2} \varphi M_{N\left(\frac{1}{2}, 0\right)}^{0}+\sin ^{2} \varphi M_{N\left(\frac{1}{2}, 0\right)}^{0}\right)
$$

which is our basic result here.

\section{Result and Discussion}

Inserting our simplifying assumption that all the "current nucleon" masses are equal, one finds the final result

$$
\Sigma_{\pi N}=\left(1+\frac{16}{3} \sin ^{2} \theta\right) M_{N}^{0} .
$$

Note that the enhancement term $\frac{16}{3} \sin ^{2} \theta$ is due to the factor $\frac{41+16}{9}=\frac{19}{3} \approx 6.33$ appearing in Eq. (․] $)$ of the $\left[\left(\mathbf{1}, \frac{\mathbf{1}}{\mathbf{2}}\right) \oplus\left(\frac{\mathbf{1}}{\mathbf{2}}, \mathbf{1}\right)\right]$ chiral multiplet, which, in turn, is due to the presence of the iso-spurion $(2 \times 4)$ matrices $T^{i}$, that are equivalent to the $\mathrm{SU}(2)$ Clebsch-Gordan coefficients $\left\langle\frac{3}{2} I_{3}(\Delta) \mid 1 I_{3}(i) \frac{1}{2} I_{3}(N)\right\rangle$. Thus, the enhancement factor $\frac{19}{3}$ in Eq. (R.J) and consequently also the $\frac{16}{3} \sin ^{2} \theta$ in Eq. (B.J), are of $S U_{L}(2) \times S U_{R}(2)$ algebraic origin. This shows that, irrespective of the specific value of the chiral mixing angle $\theta$, there is room for improvement of the $\Sigma_{\pi N}$ predictions within the chiral $S U_{L}(2) \times S U_{R}(2)$ algebra approach.

The relevant chiral mixing angle $\theta$ has been extracted in Refs. [15], [6, [17, 118] as $\frac{8}{3} \sin ^{2} \theta=$ $g_{A}^{(0)}+g_{A}^{(3)}$, a function of $g_{A}^{(3)}$, and the flavor-singlet axial coupling $g_{A}^{(0)}=0.28 \pm 0.16$ [22], or $g_{A}^{(0)}=0.33 \pm 0.03 \pm 0.05$ [D23]. Here we have taken the values of current quark masses from PDG2012 [R4]: $m_{u}^{0}=2.3 \times 1.35 \mathrm{MeV}$ and $m_{d}^{0}=4.8 \times 1.35 \mathrm{MeV}$, yielding $\frac{1}{2}\left(m_{u}^{0}+m_{d}^{0}\right) \approx 4.73$ $\mathrm{MeV}$, substantially lower than before (cf. $7.6 \mathrm{MeV}$ in Ref. [25]), and inserted them into the current nucleon mass to find $M_{N}^{0}=\frac{3}{2}\left(m_{u}^{0}+m_{d}^{0}\right) \approx 14.2 \mathrm{MeV}$ and $\Sigma_{\pi N}=59.5 \pm 2.3 \mathrm{MeV}$, with $g_{A}^{(0)}=$ $0.33 \pm 0.03 \pm 0.05$ [[2]], or $\Sigma_{\pi N}=58.0 \pm 4.5 \mathrm{MeV}$, with $g_{A}^{(0)}=0.28 \pm 0.16$ [22] , in fair agreement with the "observed" $\Sigma_{\pi N}$ value range (55 - 75) MeV, see Ref. [四].

The above result Eq. (B. $)$ ought to be viewed as a lower bound on the "true" $\Sigma_{\pi N}$ value, as we have assumed that all current nucleon masses $M_{N_{i}}^{0}$ equal three times the isospin-averaged current quark mass $\bar{m}_{q}^{0}$, which is appropriate only when all chiral components of the nucleon consist of three-quark fields. That, however, condition is not necessary (because there are $q^{4} \bar{q}$ baryon fields that belong to the same chiral multiplets [ [2]], and such fields would have a larger current mass $\left(M_{N^{\prime}}^{0}=5 \bar{m}_{q}^{0}\right.$, that would consequently lead to a higher value of $\left.\Sigma_{\pi N}\right)$, but merely a sufficient one, as all of these chiral multiplets exist as bi-local three-quark fields [20]). 
In summary, the "observed" $\Sigma_{\pi N}$ term values $(\geq 55 \mathrm{MeV})$ have often been interpreted as a sign of a substantial $s \bar{s}$ content of the nucleon. Here we have shown that values of $\Sigma_{\pi N} \geq 55 \mathrm{MeV}$ are readily obtained in the chiral-mixing approach without any strangeness degrees of freedom in the nucleon, as a natural consequence of the substantial chiral $(6,3)=[(\mathbf{6}, \mathbf{3}) \oplus(\mathbf{3}, \mathbf{6})] \rightarrow\left(1, \frac{1}{2}\right)$ multiplet component. The precise value of $\Sigma_{\pi N}$ is a linear function of the sum of the flavor-singlet $g_{A}^{(0)}$, and the isovector $g_{A}^{(3)}$ axial coupling of the nucleon.

\section{References}

[1] T. P. Cheng, Phys. Rev. D 13, 2161 (1976).

[2] J. F. Donoghue and C. R. Nappi, Phys. Lett. B 168, 105 (1986).

[3] R. L. Jaffe and C. L. Korpa, Comments Nucl. Part. Phys. 17, 163 (1987).

[4] B. Borasoy and U. -G. Meissner, Annals Phys. 254, 192 (1997); M. M. Pavan, I. I. Strakovsky, R. L. Workman and R. A. Arndt, PiN Newslett. 16, 110 (2002); G. E. Hite, W. B. Kaufmann and R. J. Jacob, Phys. Rev. C 71, 065201 (2005).

[5] A. Acha et al. [HAPPEX Collaboration], Phys. Rev. Lett. 98, 032301 (2007); S. Baunack, K. Aulenbacher, D. Balaguer Rios, L. Capozza, J. Diefenbach, B. Glaser, D. von Harrach and Y. Imai et al., Phys. Rev. Lett. 102, 151803 (2009); D. Androic et al. [G0 Collaboration], Phys. Rev. Lett. 104, 012001 (2010).

[6] V. Dmitrašinović and S. J. Pollock, Phys. Rev. C 52, 1061 (1995).

[7] J. R. Ellis, K. A. Olive and C. Savage, Phys. Rev. D 77, 065026 (2008).

[8] I. S. Gerstein and B. W. Lee, Phys. Rev. Lett. 16, 1060 (1966).

[9] H. Harari, Phys. Rev. Lett. 16, 964 (1966).

[10] H. Harari, Phys. Rev. Lett. 17, 56 (1966).

[11] H. -X. Chen, V. Dmitrašinović and A. Hosaka, Phys. Rev. C 85, 055205 (2012).

[12] V. Dmitrašinović and F. Myhrer, Phys. Rev. C 61, 025205 (2000).

[13] R. Dashen, Phys. Rev. 183, 1245 (1969); R. Dashen and M. Weinstein, Phys. Rev. 183, 1261 (1969), ibid. 188, 2330 (1969).

[14] E. Reya, Rev. Mod. Phys. 46, 545 (1974).

[15] V. Dmitrašinović, A. Hosaka and K. Nagata, Mod. Phys. Lett. A 25, 233 (2010).

[16] V. Dmitrašinović, K. Nagata, and A. Hosaka, Mod. Phys. Lett. A 23, 2381 (2008).

[17] K. Nagata, A. Hosaka and V. Dmitrašinović, Eur. Phys. J. C 57 (2008) 557.

[18] K. Nagata, A. Hosaka and V. Dmitrašinović, Phys. Rev. Lett. 101, 092001 (2008).

[19] H. -X. Chen, V. Dmitrašinović and A. Hosaka, Phys. Rev. D 81, 054002 (2010).

[20] V. Dmitrašinović and H. -X. Chen, Eur. Phys. J. C 71, 1543 (2011).

[21] H. -X. Chen, V. Dmitrašinović and A. Hosaka, Phys. Rev. D 83, 014015 (2011).

[22] B. W. Filippone and X. -D. Ji, Adv. Nucl. Phys. 26, 1 (2001).

[23] W. Vogelsang, J. Phys. G 34, S149 (2007).

[24] J. Beringer et al. [Particle Data Group Collaboration], Phys. Rev. D 86, 010001 (2012).

[25] C. Caso et al. [Particle Data Group Collaboration], Eur. Phys. J. C 3, 1 (1998). 